B. Charbonnel · G. Schernthaner $\cdot$ P. Brunetti •

D. R. Matthews $\cdot$ R. Urquhart - M. H. Tan •

M. Hanefeld

\title{
Long-term efficacy and tolerability of add-on pioglitazone therapy to failing monotherapy compared with addition of gliclazide or metformin in patients with type 2 diabetes
}

Received: 18 November 2004 / Accepted: 12 February 2005 / Published online: 12 May 2005

(C) Springer-Verlag 2005

\begin{abstract}
Aims/hypothesis: The aim of this analysis was to examine the long-term effects of pioglitazone or gliclazide addition to failing metformin monotherapy and pioglitazone or metformin addition to failing sulphonylurea monotherapy in patients with type 2 diabetes. Methods: Two 2-year, randomised, multicentre trials were performed in patients with inadequately controlled type 2 diabetes $\left(\mathrm{HbA}_{1} \mathrm{c} 7.5\right.$ $11 \%$ inclusive), who were receiving either metformin or
\end{abstract}

B. Charbonnel $(\triangle)$

Endocrinology Clinic, Hotel Dieu,

Place Alexis Ricordeau,

44093 Nantes Cedex 1, France

e-mail: Bernard.Charbonnel@univ-nantes.fr

Tel.: +33-2-40083642

Fax: +33-2-40083079

G. Schernthaner

Department of Medicine 1,

Rudolfstiftung Hospital,

Vienna, Austria

P. Brunetti

Department of Internal Medicine and Metabolic Diseases, University of Perugia,

Perugia, Italy

D. R. Matthews

Oxford Centre for Diabetes,

Endocrinology and Metabolism,

Churchill Hospital,

Oxford, UK

R. Urquhart

Takeda Europe R\&D Centre,

London, UK

M. H. Tan

Eli Lilly and Company,

Indianapolis, IN, USA

M. Hanefeld

Centre for Clinical Studies,

GWT Technical University,

Dresden, Germany a sulphonylurea at $\geq 50 \%$ of the maximum recommended dose or at the maximum tolerated dose. In the first study, patients on metformin received add-on therapy with pioglitazone ( $15-45 \mathrm{mg} / \mathrm{day}, n=317)$ or gliclazide $(80-320 \mathrm{mg} /$ day, $n=313)$. In the second study, patients on sulphonylurea therapy were randomised to receive add-on therapy with either pioglitazone (15-45 mg/day, $n=319)$ or metfor$\min (850-2,550 \mathrm{mg} / \mathrm{day}, n=320) . \mathrm{HbA}_{1} \mathrm{c}$, fasting plasma glucose, insulin and lipids were investigated. Results: At week 104, the mean reduction from baseline in $\mathrm{HbA}_{1} \mathrm{c}$ was $0.89 \%$ for pioglitazone and $0.77 \%$ for gliclazide addition to metformin $(p=0.200)$. There was a statistically significant between-group difference for the change in mean fasting plasma glucose at week $104(-1.8 \mathrm{mmol} / \mathrm{l}$ for pioglitazone vs $-1.1 \mathrm{mmol} / \mathrm{l}$ for gliclazide, $p<0.001)$. There were no significant differences in changes from baseline in glycaemic parameters for pioglitazone compared with metformin addition to sulphonylurea therapy. Whether added to metformin or sulphonylurea, pioglitazone caused significantly greater decreases in triglycerides and significantly greater increases in HDL cholesterol than the comparator regimens $(p \leq 0.001)$. There were decreases in LDL cholesterol in the comparator groups and these were significantly different from the small changes observed with pioglitazone $(p<0.001)$. All treatment regimens were well tolerated. There were weight increases of $2.5 \mathrm{~kg}$ and 3.7 $\mathrm{kg}$ in the pioglitazone and $1.2 \mathrm{~kg}$ in the gliclazide add-on groups, and there was a mean decrease of $1.7 \mathrm{~kg}$ in the metformin add-on group. Conclusions/interpretation: As add-on therapy to existing sulphonylurea or metformin therapy, pioglitazone improved glycaemic control and this improvement was sustained over 2 years. Furthermore, there were potential benefits in terms of improvements in specific lipid abnormalities. This could offer an advantage over the addition of other oral agents in the long-term treatment of diabetes.

Keywords Add-on · Gliclazide · Long-term · Metformin · Pioglitazone $\cdot$ Sulphonylurea $\cdot$ Sustained $\cdot$ Type 2 diabetes 


\begin{abstract}
Abbreviations AIP: atherogenic index of plasma . ALT: alanine aminotransferase - AST: aspartate aminotransferase $\cdot$ ITT: intention-to-treat $\cdot$ NOS: not otherwise specified $\cdot \operatorname{PPAR} \gamma$ : peroxisome proliferator activated receptor $\gamma \cdot \gamma \cdot$ TZD: thiazolidinedione $\cdot$

UKPDS: United Kingdom Prospective Diabetes Study . WHO: World Health Organization
\end{abstract}

\section{Introduction}

Monotherapy with metformin or sulphonylureas often improves glycaemic control in the short term. However, it is well documented that many patients receiving long-term oral glucose-lowering monotherapy experience a progressive deterioration in glycaemic control over time. Every year, approximately $5-10 \%$ of patients treated with a sulphonylurea or metformin experience "secondary failure" $[1,2]$. In the United Kingdom Prospective Diabetes Study (UKPDS), patients receiving sulphonylurea or metformin monotherapy experienced a gradual increase in $\mathrm{HbA}_{1} \mathrm{c}$ over a study period of up to 10 years [2-5]. The addition of a second agent with additive or synergistic effects is required to overcome such failures of monotherapy and to address different aspects of the pathogenesis of type 2 diabetes. The most commonly used combination regimen consists of metformin plus a sulphonylurea, and agents with novel modes of action, e.g. the thiazolidinediones (TZDs), pioglitazone and rosiglitazone, have been introduced more recently.

Several clinical trials have demonstrated the short-term beneficial effects of pioglitazone on glycaemic control, both alone [6-11] and when added as a second agent in combination therapy [12-15]. These studies have also reported that pioglitazone modulates carbohydrate and lipid metabolism, increases insulin sensitivity, affects vasculature and may improve some of the risk factors associated with the metabolic syndrome [6-15].

To evaluate the effects of oral glucose-lowering agents in combination, it is important to have studies of long duration. Few randomised controlled clinical studies have assessed the effects of combination therapy over periods greater than 1 year. Although the UKPDS assessed oral glucose-lowering treatments over a 10 -year period, the TZDs were not available when the study began. Furthermore, the UKPDS did not compare combination therapies with each other; intention-to-treat (ITT) monotherapy was compared with combination therapy randomised late in the trial. Two recent head-to-head studies evaluating the longterm efficacy, safety and tolerability of pioglitazone when added to failing metformin or a sulphonylurea in patients with type 2 diabetes are reported here. The first of these randomised, double-blind, double-dummy, parallel-group studies compared pioglitazone as add-on therapy to metformin with the widely used combination of sulphonylurea and metformin. Gliclazide was chosen as it is one of the most commonly prescribed sulphonylureas. In the second study, pioglitazone add-on therapy was compared with the addition of metformin to existing sulphonylurea therapy.
An analysis conducted at 1 year suggested that, although no significant differences in change from baseline of glycaemic parameters were observed between treatments, the pattern of glycaemia over time was different and longerterm effects might reveal differences [16, 17]. Furthermore, the 1-year analyses revealed additional benefits with pioglitazone add-on therapy over gliclazide or metformin addon therapies in terms of improvements in insulin sensitivity and specific abnormalities of diabetic dyslipidaemia. The studies were continued to 2 years and the data presented here are the results of the investigation of the glycaemic and non-glycaemic effects and whether these were sustained.

\section{Subjects and methods}

Study population Male and female patients with type 2 diabetes inadequately managed with metformin or sulphonylurea monotherapy (at $\geq 50 \%$ of the maximum recommended dose or at the maximum tolerated dose for $\geq 3$ months) were eligible for entry into the study. Inclusion criteria were: (1) age 35-75 years (inclusive); (2) $\mathrm{HbA}_{1} \mathrm{c}$ $7.5-11.0 \%$ (inclusive); (3) fasting C-peptide levels $\geq 0.50$ $\mathrm{nmol} / 1(1.5 \mathrm{ng} / \mathrm{ml})$; and (4) stable or worsening glycaemic control for $\geq 3$ months prior to screening. Patients were excluded if they had type 1 diabetes, ketoacidosis, symptomatic heart failure, acute malabsorption or chronic pancreatitis, familial polyposis coli, malignant disease in the previous 10 years, substance abuse or myocardial infarction, transient ischaemic attacks or stroke in the previous 6 months, or were pregnant. Patients previously treated with insulin, gliclazide, pioglitazone or other sulphonylureas or TZDs were not eligible for entry into the pioglitazone vs gliclazide addition to metformin study, and patients treated with insulin, metformin, pioglitazone or other TZDs were not eligible for entry into the pioglitazone vs metformin addition to sulphonylurea study.

Study designs The study designs have been reported in detail elsewhere $[16,17]$. Both studies were conducted in accordance with the Declaration of Helsinki and the requirements of Good Clinical Practice of the European Community. Ethics committee approval was obtained for each study site and all patients gave written informed consent prior to participation.

The first study compared the efficacy and safety of pioglitazone and gliclazide when added to existing metformin therapy in a 2-year trial conducted at 75 centres in nine European countries and Australia. Patients were randomised using block randomisation via a central telephone system (QTONE) to receive either pioglitazone $(15 \mathrm{mg} /$ day, $n=317$ ) or gliclazide ( $80 \mathrm{mg} /$ day, $n=313$ ) in addition to their usual regimen of metformin. During a 16-week forced-titration phase, the pioglitazone dose was increased in a step-wise manner to 30 and $45 \mathrm{mg}$, and the gliclazide dose to $160 \mathrm{mg}, 240 \mathrm{mg}$ (160 and $80 \mathrm{mg}$ ) and $320 \mathrm{mg}$ (160 mg twice daily), according to tolerability. No change in metformin dose from the pre-study level was allowed at any stage of the study. 
The pioglitazone vs metformin addition to sulphonylurea study was similar in design and conducted over a 2year period (the first patient was enrolled in October 2000 and the last patient completed in June 2003) at 91 centres in 12 European countries and Canada. Patients inadequately controlled with existing sulphonylurea monotherapy were randomised to add-on therapy with either pioglitazone or metformin for a 12-week forced-titration period followed by a 92 -week maintenance period. Patients received either pioglitazone (at a dose of up to $45 \mathrm{mg}$ /day) or metformin (at a dose of up to $2,550 \mathrm{mg} /$ day), plus their usual regimen of sulphonylurea. Patients initially received a dose of 15 $\mathrm{mg}$ /day of pioglitazone or $850 \mathrm{mg} /$ day of metformin, and dose levels were increased at weeks 4,8 and 12 , according to tolerability. If a patient experienced symptomatic hypoglycaemia during titration, the dose of sulphonylurea could be reduced (one-step reduction). The aim was to maintain the sulphonylurea and the study drug doses at the levels achieved at the end of the titration period; thus, in both studies, cessation of titration or a one-step down-titration was permitted only on the basis of tolerability issues or perceived risk of hypoglycaemia.

Efficacy and safety measurements In both studies the primary efficacy measure was a change in $\mathrm{HbA}_{1} \mathrm{c}$. A noninferiority analysis was performed for the primary objective. Secondary efficacy measures included changes in fasting plasma glucose, insulin and insulin precursors and lipids.

A central laboratory (Covance Central Laboratory Services SA, Geneva, Switzerland) conducted the analyses of all blood samples. $\mathrm{HbA}_{1} \mathrm{c}$ was measured using an automated ion-exchange HPLC assay (VARIANT $\mathrm{HbA}_{1} \mathrm{c}$ program; BioRad Laboratories, Hercules, CA, USA) that was standardised against the Diabetes Control and Complications Trial (DCCT) reference method. Intra-assay variability was $\leq 2.1 \%$. Serum insulin was measured using Microparticle Enzyme Immunoassay (MEIA) technology (Abbott Laboratories, Abbott Park, IL, USA) that was referenced against the World Health Organization (WHO) insulin first International Reference Preparation at $92.5 \%$ of the WHO concentration. Intra-assay variability was $\leq 6.0 \%$. Lipids were analysed by the homogeneous enzymatic colorimetric test using the Roche Diagnostics High-Performance Cholesterol method (Indianapolis, IN, USA). Intra-assay variability was $\leq 2.0 \%$ for triglycerides, $\leq 4.0 \%$ for $\mathrm{HDL}$ cholesterol and $\leq 1.4 \%$ for total cholesterol. Measurements of $\mathrm{HbA}_{1} \mathrm{c}$, fasting plasma glucose and insulin were made at baseline and weeks 4, 8, 12, 16, 24, 32, 42, 52, 62, 72, 82, 92 and 104. Lipids (triglycerides, HDL cholesterol, LDL cholesterol and total cholesterol) were measured at baseline and weeks 8, 16, 24, 32, 42, 52, 62, 72, 82, 92 and 104. LDL cholesterol was calculated using Friedewald's formula, and the atherogenic index of plasma (AIP) was calculated from the $\log _{10}$ (triglyceride: HDL cholesterol) [18]. Fasting NEFA were assessed at baseline and weeks 52 and 104 in a subgroup of the study population. Serum C-peptide and 32,33 split pro-insulin were measured at selected centres at baseline and weeks $24,42,52,72,92$ and 104.

The tolerability and safety of the therapies were assessed by: physical examination at screening and weeks 52 and 104; regular monitoring of adverse events, body weight, blood pressure and pulse rate; and standard safety laboratory tests. Clinical chemistry and haematology were performed at the central laboratory.

Statistical analysis The statistical and analytical methods have been described previously $[16,17]$. The differences in the changes in $\mathrm{HbA}_{1} \mathrm{c}$ between treatment groups were compared using an analysis of covariance model, with treatment used as the factor and baseline value as covariate. The difference between adjusted means was calculated with $95 \%$ CIs and a two-sided $t$-test $(\alpha=0.05)$ was performed. The analysis was carried out on the ITT and per-protocol populations. Other efficacy variables were analysed in a similar way. The ITT population included all patients who received at least one dose of study medication after randomisation and for whom at least one post-baseline $\mathrm{HbA}_{1} \mathrm{c}$ value was available. The per-protocol population included all patients who received at least one dose of study medication after randomisation and for whom a value for $\mathrm{HbA}_{1} \mathrm{c}$ was available at week 72 or later. A $p$ value less than or equal to 0.05 was considered significant for the primary and secondary analyses. Since the triglyceride data were not normally distributed, the lipid data were logarithmically transformed. Descriptive statistics were used to summarise demographic and baseline characteristics, adverse events and changes in body weight and laboratory variables.

\section{Results}

\section{Baseline characteristics}

The baseline data have been published previously $[16,17]$. In both studies the treatment groups have similar baseline characteristics, and the groups were generally well matched in terms of sex, age, BMI and duration of diabetes (Table 1). Comparison of the two study populations revealed that patients in the sulphonylurea add-on study had a longer duration of diabetes and patients in the metformin add-on study were slightly heavier. Figure $1 \mathrm{a}$ and $\mathrm{b}$ show the patient disposition and reasons for discontinuation of study medication. Approximately $75 \%$ of the patients enrolled completed the 104-week studies.

At the end of the dose-titration period, $70 \%$ of patients receiving pioglitazone add-on to metformin had been titrated to the maximum pioglitazone dose of $45 \mathrm{mg} /$ day (mean dose $39 \mathrm{mg} /$ day), whereas $33 \%$ of patients in the gliclazide arm had been titrated to the maximum gliclazide dose of $320 \mathrm{mg} /$ day (mean dose $212 \mathrm{mg} /$ day).

Of the 952 patients screened for the pioglitazone vs metformin addition to sulphonylurea study, 639 patients were treated with pioglitazone $(n=319)$ or metformin $(n=$ 
Table 1 Demographic and baseline characteristics

\author{
The data shown are means \pm SD \\ or $n(\%)$ \\ ${ }^{\mathrm{a}}$ To convert $\mathrm{mmol} / \mathrm{l}$ to $\mathrm{mg} / \mathrm{dl}$ \\ divide by 0.0555 \\ ${ }^{\mathrm{b}}$ To convert $\mathrm{mmol} / \mathrm{l}$ to $\mathrm{mg} / \mathrm{dl}$ \\ divide by 0.0113 \\ ${ }^{\mathrm{c}}$ To convert $\mathrm{mmol} / \mathrm{l}$ to $\mathrm{mg} / \mathrm{dl}$ \\ divide by 0.0259
}

\begin{tabular}{|c|c|c|c|c|}
\hline \multirow[t]{2}{*}{ Variable } & \multicolumn{2}{|c|}{ Add-on to metformin } & \multicolumn{2}{|c|}{ Add-on to sulphonylurea } \\
\hline & $\begin{array}{l}\text { Pioglitazone } \\
(n=317)\end{array}$ & $\begin{array}{l}\text { Gliclazide } \\
(n=313)\end{array}$ & $\begin{array}{l}\text { Pioglitazone } \\
(n=319)\end{array}$ & $\begin{array}{l}\text { Metformin } \\
(n=320)\end{array}$ \\
\hline \multicolumn{5}{|l|}{ Sex } \\
\hline Male & $161(50.8 \%)$ & $154(49.2 \%)$ & $171(53.6 \%)$ & $175(54.7 \%)$ \\
\hline Female & $156(49.2 \%)$ & $159(50.8 \%)$ & $148(46.4 \%)$ & $145(45.3 \%)$ \\
\hline \multicolumn{5}{|l|}{ Race } \\
\hline Caucasian & $315(99.4 \%)$ & $313(100 \%)$ & $317(99.4 \%)$ & $315(98.4 \%)$ \\
\hline Black & 0 & 0 & $2(0.6 \%)$ & $3(0.9 \%)$ \\
\hline Other & $2(0.6 \%)$ & 0 & 0 & $2(0.6 \%)$ \\
\hline Age (years) [range] & $56 \pm 9.2[35-74]$ & $57 \pm 9.0[34-75]$ & $60 \pm 8.8[36-75]$ & $60 \pm 8.0[36-75]$ \\
\hline BMI $\left(\mathrm{kg} / \mathrm{m}^{2}\right)$ [range] & $32.6 \pm 5.0[23-52]$ & $32.6 \pm 5.8[20-59]$ & $30.2 \pm 4.4[21-45]$ & $30.0 \pm 4.6[19-46]$ \\
\hline $\begin{array}{l}\text { Duration of diabetes } \\
\text { (years) [range] }\end{array}$ & $5.8 \pm 5.1[0.2-30.9]$ & $5.5 \pm 5.1[0.2-34.7]$ & $7.0 \pm 5.6[0.2-30.7]$ & $7.1 \pm 5.6[0.3-31.7]$ \\
\hline $\mathrm{HbA}_{1} \mathrm{c}(\%)$ & $8.71 \pm 1.00$ & $8.53 \pm 0.89$ & $8.82 \pm 0.98$ & $8.80 \pm 0.98$ \\
\hline $\begin{array}{l}\text { Fasting plasma } \\
\text { glucose }(\mathrm{mmol} / \mathrm{l})^{\mathrm{a}}\end{array}$ & $11.8 \pm 3.1$ & $11.3 \pm 2.6$ & $11.8 \pm 2.7$ & $12.0 \pm 2.9$ \\
\hline $\begin{array}{l}\text { Triglycerides } \\
(\mathrm{mmol} / \mathrm{l})^{\mathrm{b}}\end{array}$ & $2.90 \pm 1.94$ & $2.78 \pm 1.89$ & $2.47 \pm 1.69$ & $2.38 \pm 1.72$ \\
\hline $\begin{array}{l}\text { HDL cholesterol } \\
(\mathrm{mmol} / \mathrm{l})^{\mathrm{c}}\end{array}$ & $1.10 \pm 0.25$ & $1.09 \pm 0.23$ & $1.09 \pm 0.24$ & $1.11 \pm 0.27$ \\
\hline $\begin{array}{l}\text { LDL cholesterol } \\
(\mathrm{mmol} / \mathrm{l})^{\mathrm{c}}\end{array}$ & $3.34 \pm 0.98$ & $3.28 \pm 0.93$ & $3.57 \pm 0.86$ & $3.58 \pm 0.92$ \\
\hline
\end{tabular}

320 ) in addition to their sulphonylurea therapy. At the end of the dose-titration period, $62 \%$ of patients in the pioglitazone group were receiving the maximum dose of 45 $\mathrm{mg}$ (mean dose of $37 \mathrm{mg} /$ day), and $55 \%$ of those in the metformin group were receiving the maximum dose of $2,550 \mathrm{mg} /$ day (mean dose $2,074 \mathrm{mg} /$ day).

\section{Glycaemic control}

The 1-year efficacy and safety data have been reported previously $[16,17]$. All data are from the ITT population unless otherwise specified.

Pioglitazone vs gliclazide addition to metformin study The differences in the changes in $\mathrm{HbA}_{1} \mathrm{c}$ from baseline to week 52 were not statistically significant between the two groups, with decreases of $0.99 \%$ and $1.01 \%$ observed with pioglitazone add-on therapy to metformin and gliclazide addon to metformin, respectively (adjusted mean difference $0.02,95 \%$ CI -0.15 to $0.19, p=0.837)$. The differences in the changes from baseline among the ITT population were not statistically significant at week $104(0.89 \%$ and $0.77 \%$ with pioglitazone and gliclazide, respectively, $p=0.200$; Fig. 2a); however, there was a statistically significant between-group difference in $\mathrm{HbA}_{1} \mathrm{c}$ reduction at week 104 in patients treated for a minimum of 18 months (perprotocol population; $1.07 \%$ and $0.76 \%$ with pioglitazone and gliclazide, respectively, $p=0.003$ ). At week 104, the proportion of patients who had achieved a target $\mathrm{HbA}_{1} \mathrm{c}$ level of $<7.0 \%$ was $30.6 \%$ when pioglitazone was added to metformin and $25.2 \%$ for gliclazide addition to metformin $(p=0.128)$.
Mean fasting plasma glucose values revealed that, despite comparable reductions in fasting plasma glucose at week 52 , there was a statistically significant difference between the pioglitazone add-on to metformin group and the gliclazide add-on to metformin group at week 104 ( -1.8 vs $-1.1 \mathrm{mmol} / 1, p<0.001$; Fig. $2 \mathrm{~b})$.

Pioglitazone vs metformin addition to sulphonylurea study For patients receiving add-on therapy with either pioglitazone or metformin to sulphonylurea, there were no significant between-treatment differences in terms of $\mathrm{HbA}_{1} \mathrm{c}$ or fasting plasma glucose. At week $104, \mathrm{HbA}_{1} \mathrm{c}$ was reduced by $1.03 \%$ with pioglitazone addition to sulphonylurea and by $1.16 \%$ with metformin addition to sulphonylurea (adjusted mean difference $-0.13,95 \% \mathrm{CI}-0.06$ to $0.31, p=0.173$; Fig. 3a). At week 104, the proportion of patients who had achieved a target $\mathrm{HbA}_{1} \mathrm{c}$ level of $<7.0 \%$ was $30.2 \%$ for pioglitazone addition to sulphonylurea and $28.4 \%$ for metformin addition to sulphonylurea $(p=0.635)$.

From baseline to week 104, fasting plasma glucose was reduced by $2.0 \mathrm{mmol} / \mathrm{l}$ in the pioglitazone add-on to sulphonylurea group and $1.9 \mathrm{mmol} / \mathrm{l}$ in the metformin add-on to sulphonylurea group (adjusted mean difference -0.02 , $95 \% \mathrm{CI}-0.6$ to $0.3, p=0.506$; Fig. $3 b$ ).

Serum lipid levels

Figure 4 shows the changes in lipid profiles (ITT population). Whether added to metformin or sulphonylurea, pioglitazone caused significantly greater decreases in triglyceride levels and significantly greater increases in HDL cholesterol levels $(p \leq 0.001$ for all between-group compar- 
Fig. 1 Patient disposition in the pioglitazone vs gliclazide addition to metformin study (a) and the pioglitazone vs metformin addition to sulphonylurea study (b)
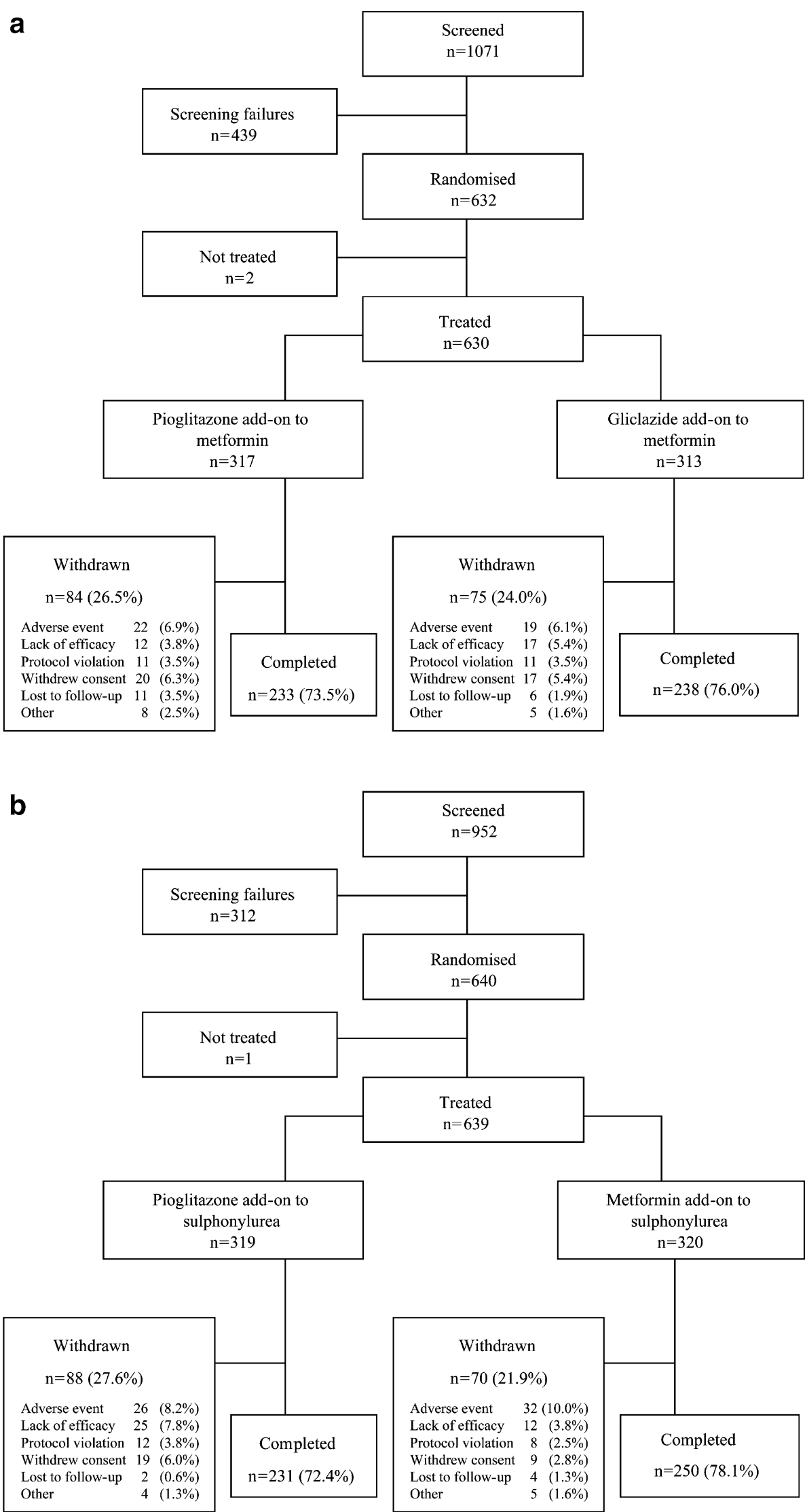
Fig. 2 Time course of mean changes \pm SEM from baseline to last value (last observation carried forward analysis) for $\mathrm{HbA}_{1} \mathrm{c}$ (a) and fasting plasma glucose (b) in the ITT population. To convert $\mathrm{mmol} / \mathrm{l}$ to $\mathrm{mg} / \mathrm{dl}$ divide by 0.0555 . Closed circles, pioglitazone add-on to metformin; open squares, gliclazide add-on to metformin a

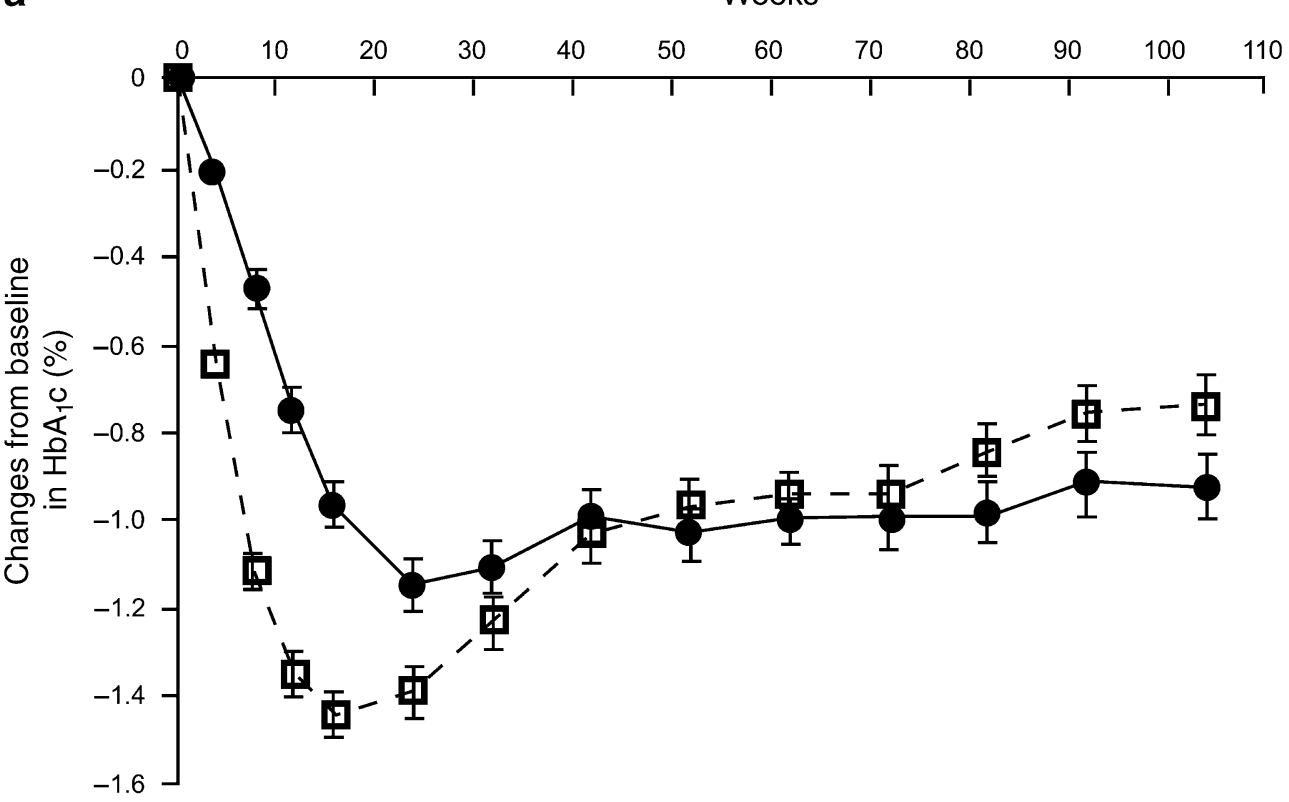

b

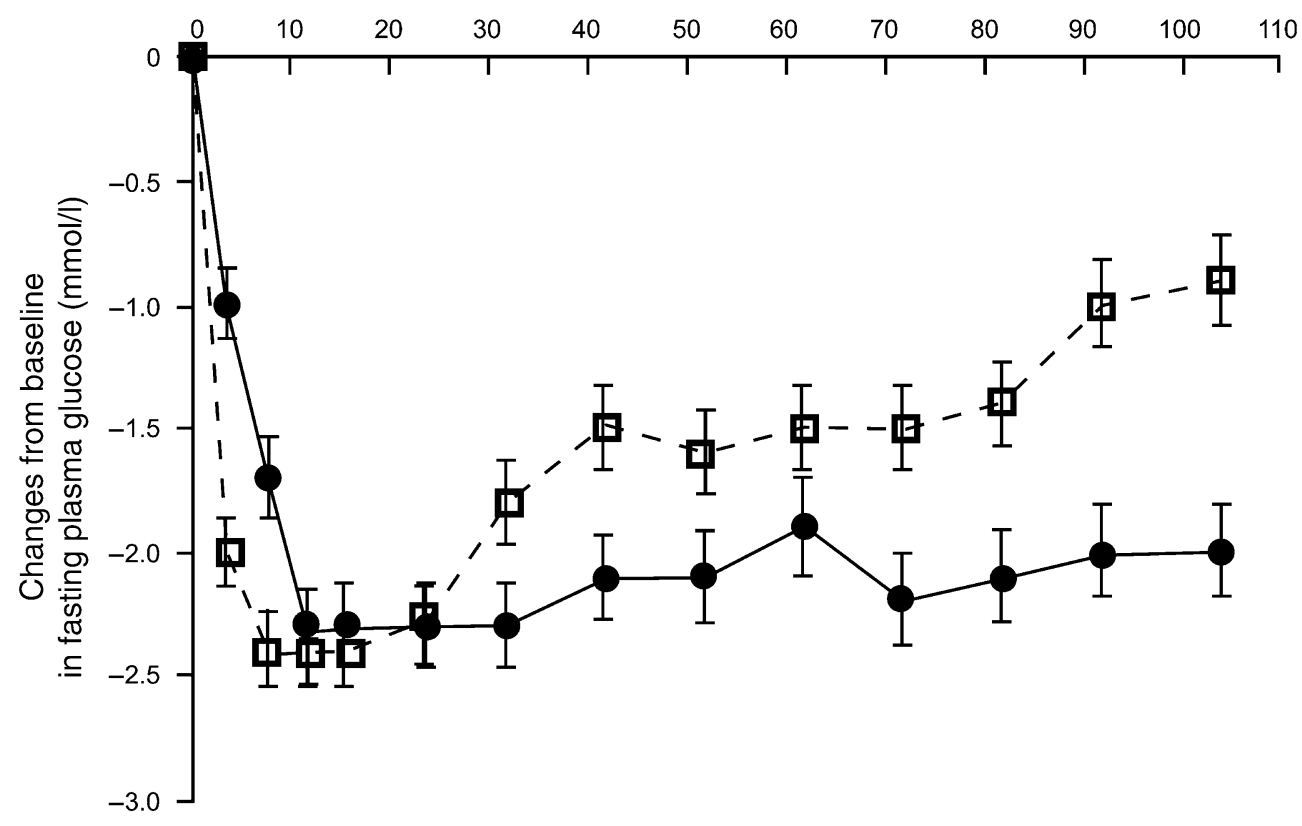

isons). Gliclazide addition to metformin and metformin addition to sulphonylurea decreased LDL cholesterol and total cholesterol, whereas there was little change in these parameters in the pioglitazone groups (increases or decreases $\leq 5 \%$ for LDL cholesterol and $\leq 1 \%$ for total cholesterol). The differences between groups were statistically significant ( $p<0.001$ for LDL cholesterol, $p \leq 0.005$ for total cholesterol). Among patients who received lipid-lowering therapy at baseline $(18.3 \%$ of those enrolled in the pioglitazone vs gliclazide addition to metformin study and $15.3 \%$ of those in the pioglitazone vs metformin addition to sulphonylurea study), the doses remained stable throughout the study, and the effects of add-on therapies on lipid levels in this population were comparable to those indicated by the ITT analysis.

When added to either metformin or sulphonylurea therapy, pioglitazone caused significantly greater decreases in the AIP, a surrogate marker of LDL particle size, than the comparators $(-0.46$ for add-on to metformin vs -0.14 for gliclazide addition $[p<0.001]$ and -0.38 for add-on to sulphonylurea vs -0.23 for metformin addition $[p<0.001]$ ).

Mean fasting plasma NEFA levels were decreased by $0.11 \mathrm{mmol} / \mathrm{l}$ in the pioglitazone add-on to metformin group $(n=37)$ and by $0.04 \mathrm{mmol} / 1$ in the gliclazide add-on to metformin group $(n=64 ; p=0.046$ for between-group difference), and by $0.08 \mathrm{mmol} / \mathrm{l}$ in the pioglitazone add-on to 
Fig. 3 Time course of mean changes \pm SEM from baseline to last value (last observation carried forward analysis) for $\mathrm{HbA}_{1} \mathrm{c}$ (a) and fasting plasma glucose (b) in the ITT population. To convert $\mathrm{mmol} / \mathrm{l}$ to $\mathrm{mg} / \mathrm{dl}$ divide by 0.0555 . Open circles, pioglitazone add-on to sulphonylurea; closed triangles, metformin add-on to sulphonylurea a

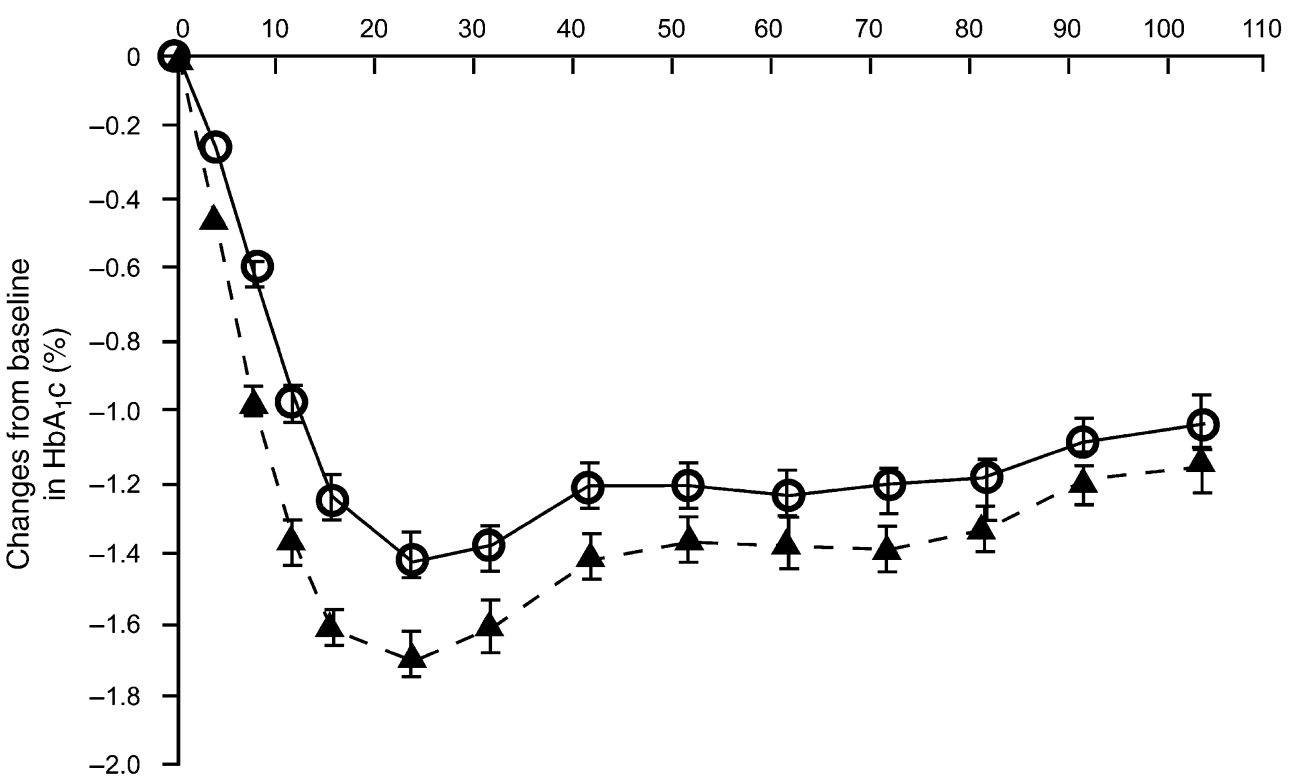

b

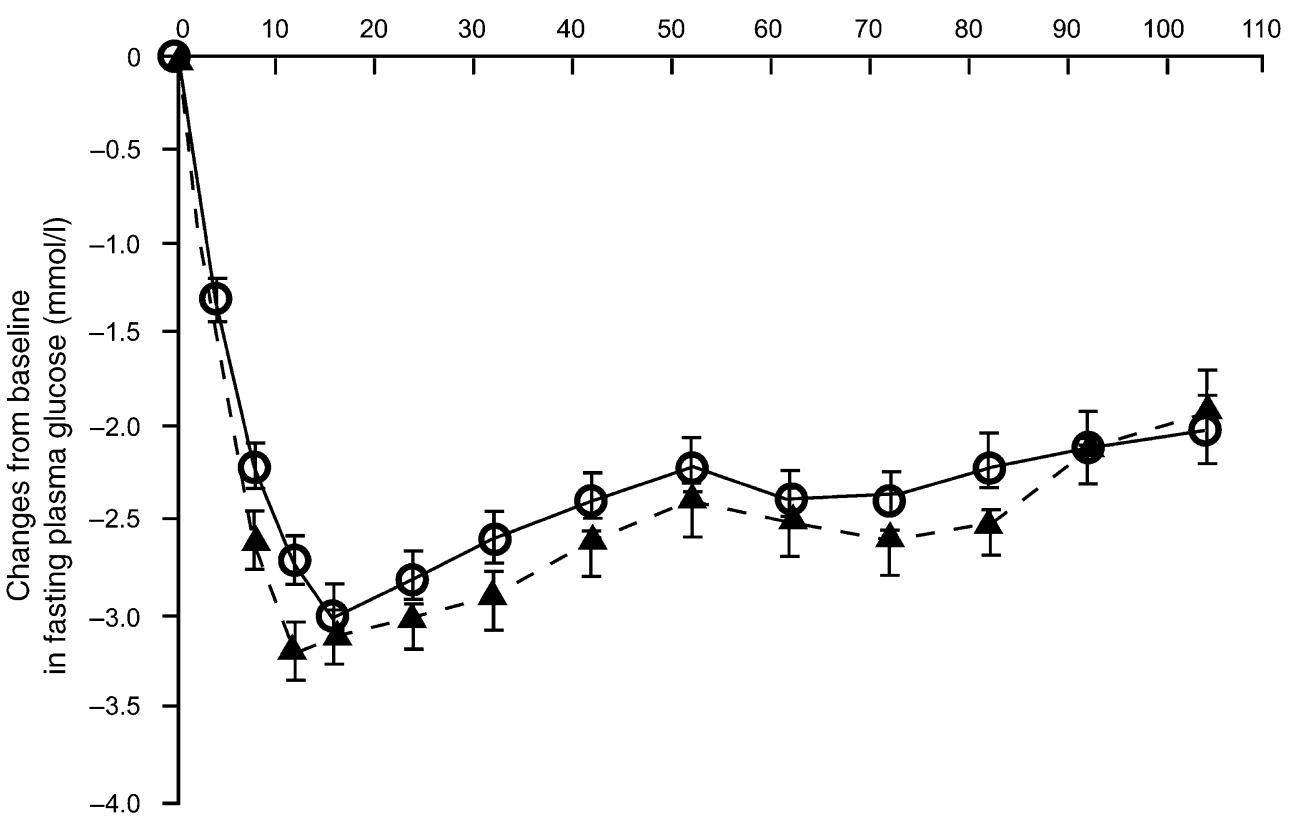

sulphonylurea group ( $n=85$ ) compared with $0.03 \mathrm{mmol} / \mathrm{l}$ in the metformin add-on to sulphonylurea group $(n=99 ; p=$ 0.053 for between-group difference).

Serum insulin and insulin precursors

Pioglitazone caused sustained decreases in fasting serum insulin whether added to metformin or sulphonylurea, whereas there were increases in the gliclazide add-on to metformin group. In the metformin add-on to sulphonylurea group, the decreases in fasting serum insulin observed at week 52 [16] had risen back towards baseline values by week 104 (Table 2). The difference in the change in serum insulin with pioglitazone vs gliclazide addition to metformin group was statistically significant $(p<0.001)$.

Insulin precursors were measured at selected centres. The addition of pioglitazone to metformin $(n=168)$ caused decreases in levels of intact pro-insulin and 32,33 split proinsulin, whereas the addition of gliclazide to metformin caused a small increase in intact pro-insulin $(n=169)$ and a small decrease in mean 32,33 split pro-insulin $(n=166)$. The difference between treatments was statistically significant for both variables $(p<0.001$; Table 2$)$. In the pioglitazone vs metformin addition to sulphonylurea study, both pioglitazone and metformin caused decreases from baseline in intact pro-insulin ( $n=166$ and $n=155$ for pioglitazone and metformin, respectively) and 32,33 split pro- 
Fig. 4 Changes from baseline to last value (last observation carried forward analysis) for triglycerides, HDL cholesterol and LDL cholesterol in the ITT population. The treatments were compared using adjusted mean ratios from baseline (pioglitazone/metformin) with baseline values as covariates and based on logarithmically transformed data. White bars, pioglitazone (pio) add-on groups; hatched bars, gliclazide (glic) add-on group; black bars, metformin (met) add-on group. To convert $\mathrm{mmol} / \mathrm{l}$ to $\mathrm{mg} / \mathrm{dl}$, divide by 0.0113 for triglycerides and by 0.0259 for HDL cholesterol and LDL cholesterol

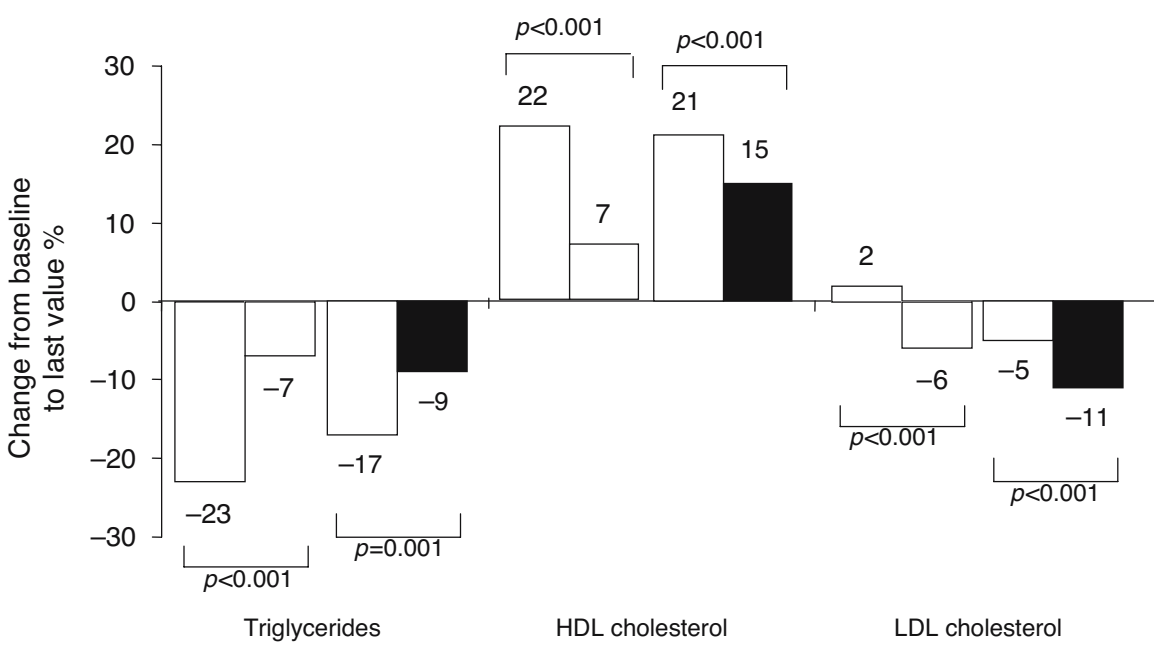

\begin{tabular}{|lcccccccccccc|}
\hline & \multicolumn{4}{c}{$\mathrm{mmol} / \mathrm{l}$} & \multicolumn{1}{c}{$\mathrm{mmol} / \mathrm{l}$} & \multicolumn{4}{c|}{$\mathrm{mmol} / \mathrm{l}$} \\
& pio & glic & pio & met & pio & glic & pio & met & pio & glic & pio & met \\
Baseline & 2.90 & 2.78 & 2.46 & 2.38 & 1.10 & 1.09 & 1.09 & 1.11 & 3.34 & 3.28 & 3.57 & 3.58 \\
Week 104 & 2.16 & 2.58 & 1.96 & 2.12 & 1.35 & 1.17 & 1.32 & 1.27 & 3.41 & 3.08 & 3.44 & 3.21 \\
95\%CI & $-0.73,-0.35$ & $-0.41,-0.06$ & $0.13,0.20$ & $0.04,0.11$ & $0.16,0.38$ & $0.11,0.36$ \\
$p$-value & $<0.001$ & 0.008 & $<0.001$ & $<0.001$ & & $<0.001$ & $<0.001$ \\
\hline
\end{tabular}

Table 2 Mean changes from baseline to week 104 in fasting insulin levels, intact pro-insulin and 32,33 split pro-insulin

${ }^{\text {a }}$ To convert $\mathrm{pmol} / 1$ to $\mu \mathrm{IU} / \mathrm{ml}$ divide by 6.945

\begin{tabular}{|c|c|c|c|c|}
\hline & \multicolumn{2}{|l|}{ Add-on to metformin } & \multicolumn{2}{|c|}{ Add-on to sulphonylurea } \\
\hline & Pioglitazone & Gliclazide & Pioglitazone & Metformin \\
\hline Fasting serum insulin $(\mathrm{pmol} / 1)^{\mathrm{a}}$ & -18.1 & 13.2 & -7.6 & -1.4 \\
\hline Adjusted difference $(95 \% \mathrm{CI})$ & $-31.3(-41.0$ to -22.2$)$ & & $-5.6(-12.5$ to 0.7$)$ & \\
\hline$p$ value & $p<0.001$ & & $p=0.075$ & \\
\hline Intact pro-insulin $(\mathrm{pmol} / \mathrm{l})^{\mathrm{a}}$ & -8.3 & 1.5 & -7.6 & -9.3 \\
\hline Adjusted difference $(95 \% \mathrm{CI})$ & $-9.8(-12.5$ to -7.1$)$ & & $1.7(-0.6$ to 4.0$)$ & \\
\hline$p$ value & $p<0.001$ & & $p=0.149$ & \\
\hline 32,33 split pro-insulin $(\mathrm{pmol} / \mathrm{l})^{\mathrm{a}}$ & -9.3 & -3.2 & -8.6 & -9.5 \\
\hline Adjusted difference $(95 \% \mathrm{CI})$ & $-6.1(-8.5$ to -3.8$)$ & & $0.9(-1.2$ to 3.0$)$ & \\
\hline$p$ value & $p<0.001$ & & $p=0.404$ & \\
\hline
\end{tabular}

Table 3 Adverse events known to occur in patients treated with sulphonylureas, metformin or pioglitazone and proportion of patients withdrawing due to them

\begin{tabular}{|c|c|c|c|c|}
\hline & \multicolumn{2}{|l|}{ Add-on to metformin } & \multicolumn{2}{|c|}{ Add-on to sulphonylurea } \\
\hline & Pioglitazone $(n=317)$ & Gliclazide $(n=313)$ & Pioglitazone $(n=319)$ & Metformin $(n=320)$ \\
\hline Symptoms compatible with hypoglycaemia & $7(2.2 \%)$ & $36(11.5 \%)$ & $36(11.3 \%)$ & $50(15.6 \%)$ \\
\hline GI disorders ${ }^{\mathrm{a}}$ & $12(3.8 \%)$ & $16(5.1 \%)$ & $20(6.3 \%)$ & $62(19.4 \%)$ \\
\hline Congestive heart failure ${ }^{b}$ & $5(1.6 \%)$ & $2(0.6 \%)$ & $2(0.6 \%)$ & $3(0.9 \%)$ \\
\hline Oedema $^{c}$ & $24(7.6 \%)$ & $11(3.5 \%)$ & $34(10.7 \%)$ & $9(2.8 \%)$ \\
\hline
\end{tabular}

The data shown are $n(\%)$

${ }^{a}$ Includes abdominal discomfort, distension and pain; diarrhoea; flatulence; frequent bowel movements; gastrointestinal pain; loose stools; watery stools

b Acute, aggravated, congestive, chronic and not otherwise specified (NOS) cardiac failure; left ventricular failure; aggravated congestive cardiac failure; acute, aggravated and NOS pulmonary oedema

${ }^{\mathrm{c}}$ Gravitational, localised, neck, abdomen NOS, peripheral, orbital, pitting and NOS oedema

insulin when added to sulphonylurea $(n=164$ and $n=154$ for pioglitazone and metformin, respectively). There were no significant differences between the two groups for either variable (Table 2).
Safety and tolerability assessment

All four treatment regimens were well tolerated and the majority of adverse events were mild to moderate in 
Fig. 5 Changes from baseline to last value (last observation carried forward analysis) for weight gain in the ITT population. The treatments were compared using adjusted mean ratios from baseline (pioglitazone/ metformin) with baseline values as covariates. White bars, pioglitazone add-on groups; hatched bars, gliclazide add-on group; black bars, metformin add-on group

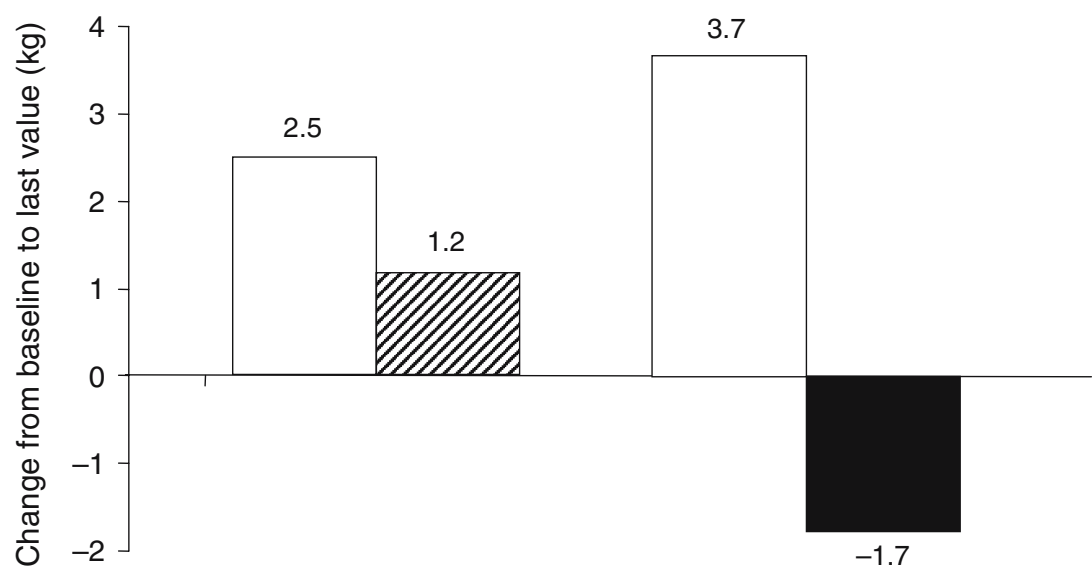

severity. There were no major differences between groups with respect to the number of adverse events, and a similar number of patients in each treatment group discontinued the add-on therapy due to adverse events, with $22(6.9 \%)$ in the pioglitazone add-on to metformin group, $21(6.7 \%)$ in the gliclazide add-on to metformin group, $28(8.8 \%)$ in the pioglitazone add-on to sulphonylurea group and 32 $(10.0 \%)$ in the metformin add-on to sulphonylurea group. As regards the individual events, there were more gastrointestinal disorders observed with metformin, more hypoglycaemia with gliclazide and more oedema with pioglitazone (Table 3).

At week 104, there were mean weight increases of $2.5 \mathrm{~kg}$ and $3.7 \mathrm{~kg}$ when pioglitazone was added to metformin and sulphonylurea, respectively, and $1.2 \mathrm{~kg}$ when gliclazide was added to metformin. A decrease in mean body weight of $1.7 \mathrm{~kg}$ was observed at week 104 when metformin was added to sulphonylurea (Fig. 5).

Liver tests, particularly alanine aminotransferase (ALT), showed consistent improvements when pioglitazone was added to metformin or sulphonylurea (Fig. 6). The addition of metformin to sulphonylurea produced smaller changes in liver parameters, and gliclazide addition to metformin resulted in marginal changes (Fig. 6).

\section{Discussion}

Short-term studies have shown pioglitazone, metformin and sulphonylureas to be effective when added as second agents to failing monotherapy $[13-15,19-21]$. These two 2-year studies provide an opportunity to compare the longer-term sustainability of glycaemic control of adding pioglitazone to metformin or sulphonylurea with the effects of adding gliclazide or metformin, respectively. We found that the combination of pioglitazone and metformin provided sustained glycaemic control and superior lipidaemic control to gliclazide with metformin, suggesting that when a patient is failing on metformin monotherapy there may be an advantage to adding pioglitazone rather than a sulphonylurea. For patients treated with pioglitazone plus sulphonylurea glycaemic control was comparable to that of
Fig. 6 Time course of mean changes from baseline to last value (last observation carried forward analysis) for ALT in the ITT population of the pioglitazone vs gliclazide addition to metformin study (a) and the pioglitazone vs metformin addition to sulphonylurea study (b). Closed circles, pioglitazone addon to metformin; open squares; gliclazide add-on to metformin; open circles, pioglitazone addon to sulphonylurea; closed triangles, metformin add-on to sulphonylurea
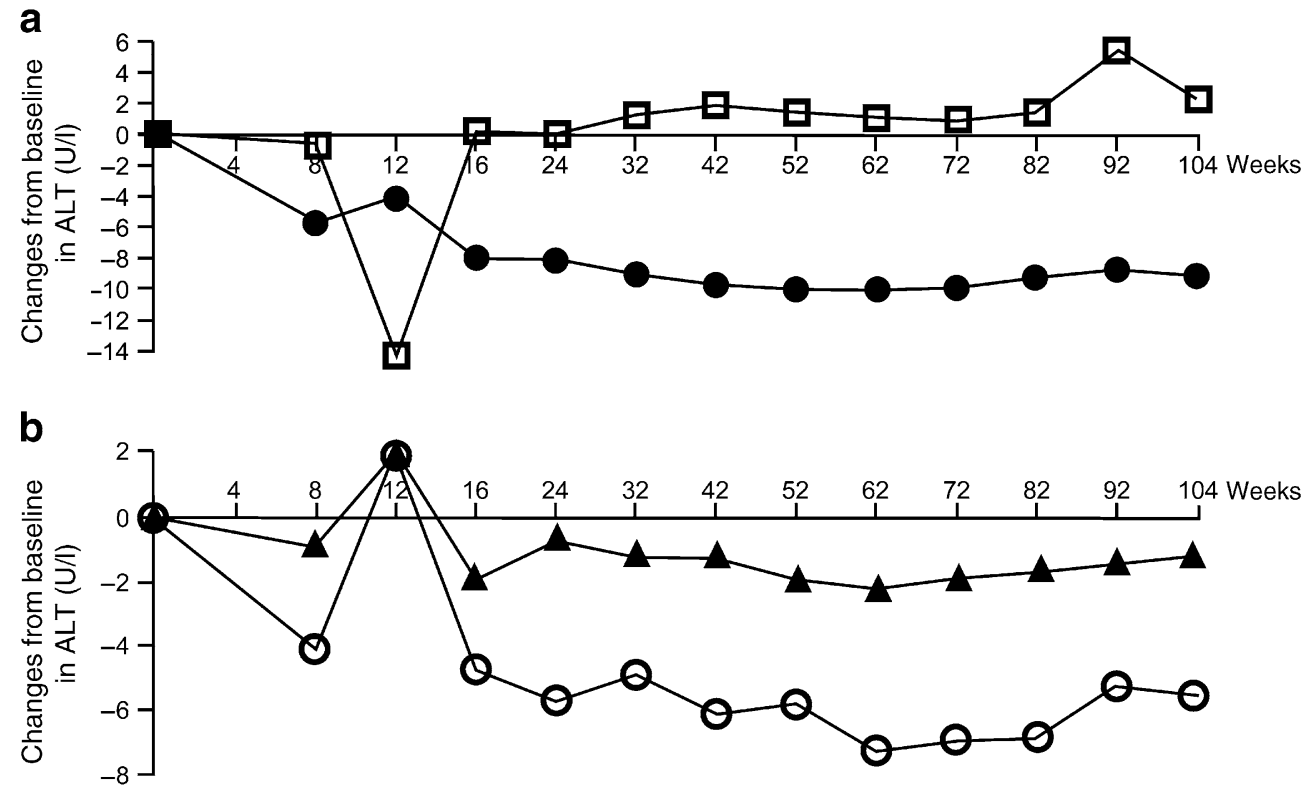
patients treated with metformin plus sulphonylurea, but there was an advantage in the pioglitazone group with respect to lipids.

Glucose-lowering therapies have been shown to reduce long-term microvascular complications [22, 23]; however, due to the natural progression of diabetes, monotherapy is often insufficient to sustain glycaemic control over the long term and a second agent is frequently needed [2-5]. For example, in the UKPDS, most of the patients needed combination therapy because of the continuous decline in beta cell function [4]. The combination of metformin (an inhibitor of hepatic glucose overproduction) plus a sulphonylurea (an insulin secretagogue) is the traditional combination option when monotherapy with either as the single agent fails [19, 24], but this is not always efficacious. Although short-term studies have shown that such combinations are initially effective for periods of less than 1 year $[20,21]$, control was reported to deteriorate over longer periods of time in the UKPDS [25]. There exists a need for agents, or combinations of agents, that can sustain glycaemic control in the longer term. From a pathophysiological point of view, the TZDs provide an alternative add-on option to failing monotherapy by virtue of their insulin-sensitising properties. The studies reported here provide the first long-term comparison of the traditional metformin plus sulphonylurea regimen with pioglitazone add-on to either metformin or a sulphonylurea.

In previous studies, $30 \mathrm{mg}$ /day of pioglitazone as add-on therapy to metformin [13] caused a mean decrease of $0.64 \%$ in $\mathrm{HbA}_{1} \mathrm{c}$ over 16 weeks compared with a $0.19 \%$ increase in $\mathrm{HbA}_{1} \mathrm{c}$ with metformin plus placebo. Seventytwo weeks of open-label pioglitazone plus metformin treatment resulted in further decreases in $\mathrm{HbA}_{1} \mathrm{c}(-1.4 \%$ from baseline) and fasting plasma glucose $(-3.5 \mathrm{mmol} / \mathrm{l}$ from baseline), indicating that glycaemic control was maintained over a period of more than 1.5 years [13]. Studies on the addition of pioglitazone to sulphonylurea, performed over periods of up to 12 months, have shown that glycaemic control is improved with this regimen compared with continued sulphonylurea monotherapy $[12,14]$.

The 2-year data reported here extend the results at 1 year $[16,17]$ : the addition of pioglitazone or a sulphonylurea to metformin and the addition of pioglitazone or metformin to a sulphonylurea were effective in decreasing $\mathrm{HbA}_{1} \mathrm{c}$ and fasting plasma glucose levels beyond those achieved by monotherapy. Interestingly, among the patients who were failing on metformin, the glycaemic control achieved with pioglitazone add-on to metformin was well sustained. At 2 years, pioglitazone induced superior decreases from baseline in fasting plasma glucose compared with gliclazide $(p \leq 0.001)$. In addition, although the difference in $\mathrm{HbA}_{1} \mathrm{c}$ changes from baseline to week 104 was not statistically significant in the ITT population, in those patients who were treated for at least 72 weeks (per-protocol population), the difference was significant in favour of pioglitazone $(p=0.003)$. In the pioglitazone vs metformin addition to sulphonylurea study, there were no statistically significant differences between the two groups in terms of the changes in $\mathrm{HbA}_{1} \mathrm{c}$ and fasting plasma glucose at 2 years.
Dyslipidaemia is an important cardiovascular risk factor. In patients with type 2 diabetes, there is a strong association between high triglyceride and low HDL cholesterol levels and cardiovascular morbidity and mortality, whereas LDL cholesterol is often normal or borderline [26-30]. Shortterm studies have reported that pioglitazone has beneficial effects on triglycerides and HDL cholesterol $[9,12,13]$. The 1-year data from the add-on studies also showed that improvements in triglyceride and HDL cholesterol levels were significantly more pronounced in the pioglitazone groups $[16,17]$. The 2-year data support these observations, with the significantly superior effects of pioglitazone over metformin and gliclazide on triglycerides and HDL cholesterol still observed at week 104. Significantly greater reductions in total cholesterol and LDL cholesterol were observed in the gliclazide add-on to metformin group and the metformin add-on to sulphonylurea group than in the respective pioglitazone add-on groups, in which only small changes (increases or decreases of $\leq 5 \%$ ) were seen for both parameters at 2 years.

Compositional alterations in LDL particles can be potentially atherogenic [31-34]. Diabetic dyslipidaemia appears to be related to the presence of smaller, dense LDL particles that are more atherogenic than larger, more buoyant particles [35]. The AIP is inversely correlated with LDL particle size [18]. Over the 2-year period reported here, the AIP was reduced by a significantly greater extent by the addition of pioglitazone to the metformin or sulphonylurea therapies than the addition of gliclazide or metformin, respectively. Taken together, these lipid data suggest that pioglitazone therapy has benefits in terms of modulating the unique dyslipidemia associated with type 2 diabetes, and that the addition of pioglitazone to failing metformin or sulphonylurea therapy offers a distinct advantage over the addition of sulphonylurea or metformin, respectively, in this regard.

At present it is not known whether these effects of pioglitazone on sustained glycaemic and lipidaemic control will translate into improved outcomes over the longer term. However, studies such as the Prospective Pioglitazone Clinical Trial in Macrovascular Events (PROactive), which was designed to evaluate the effect of pioglitazone treatment on the incidence of macrovascular comorbidity and total mortality in over 5,000 high-risk patients with type 2 diabetes, will help to establish this [36].

Another important finding was the difference between combinations with respect to insulin sensitivity and insulin processing in the beta cell. Over 2 years, pioglitazone addon therapy to either metformin or sulphonylurea caused sustained decreases in fasting insulin levels. In contrast, there was an increase in fasting insulin when gliclazide was added to metformin, and the decrease in insulin seen at week 52 when metformin was added to sulphonylurea [16] was not sustained over the 2-year treatment period, with values near to baseline levels observed at week 104. Pioglitazone add-on therapy and the addition of metformin to sulphonylurea improved insulin processing (intact pro-insulin and 32,33 split pro-insulin), while the addition of gliclazide to metformin had no effect. 
To date, there have been no clear data on the long-term $(>1$ year) safety of oral glucose-lowering agents in combination. Over the 2-year period reported here, the incidence of adverse events and the clinical laboratory values indicate that all treatment regimens were generally well tolerated. More than $70 \%$ of patients completed the studies. As expected, the main adverse events were weight gain, hypoglycaemia, oedema and gastrointestinal disorders. Rates of hypoglycaemia with gliclazide (12\%) and gastrointestinal disorders with metformin (28\%) were similar to those previously reported. Oedema $(8-11 \%)$ and weight gain $(\sim 4 \%)$ were the most common adverse events in the pioglitazone group and these have also been reported previously $[16,17]$. There have been worries concerning liver toxicity with TZDs. In the studies reported here, pioglitazone add-on caused reductions in mean levels of hepatic enzymes, while gliclazide caused either no change or small increases.

In conclusion, the data from these 2-year studies provide a strong indication that, when added to metformin, pioglitazone provides good sustainability of glycaemic control allied with an improvement over gliclazide addition to metformin in terms of insulin processing and the modulation of the unique dyslipidaemia associated with type 2 diabetes. When added to sulphonylurea, glycaemic control and improvements in insulin processing were well sustained by the addition of either pioglitazone or metformin, but improvements in diabetic dyslipidaemia were greater with pioglitazone. As expected, there was a mean weight loss in the metformin add-on group and weight increases in the pioglitazone and gliclazide add-on groups. These findings confirm that pioglitazone is a valuable alternative to the addition of metformin to failing sulphonylurea monotherapy and, in the case of addition to metformin, its potential long-term sustainability and lipid effects may make it preferable to the addition of sulphonylurea.

\section{Duality of interest statement}

B. Charbonnel has received honoraria/consulting fees for speaking engagements from the Takeda Europe R\&D Centre. G. Schernthaner is on the Board of Directors of Aventis, Servier, Astra Zeneca and Merck, and has received honoraria for speaking engagements from Aventis, Takeda, GlaxoSmithKline, Novo Nordisk, Eli Lilly and Bayer. P. Brunetti has received honoraria for lectures given in meetings organised by Eli Lilly, Novo Nordisk and Takeda. D. Matthews has received honoraria/ consulting fees for lectures and for participating in scientific boards from Novo Nordisk, GlaxoSmithKline and Takeda. The Oxford Centre for Diabetes Endocrinology and Metabolism is in a partnership that was formed initially between the NHS, the University of Oxford and Novo Nordisk, and has since been extended to include Servier Laboratories and Takeda Chemical Industries. R. Urquhart is employed by the Takeda Europe R\&D Centre, and M. Tan is employed by Eli Lilly and Company. M. Hanefeld has received consulting fees/ honoraria for lectures/consultations from Bayer, GlaxoSmithKline,
Astra Zeneca, Merck, Sharp and Dohme, Takeda, Aventis, Sanofi and local healthcare organisations in Germany.

Acknowledgements We would like to thank all the investigators who took part in this study. This work was supported by Takeda Europe R\&D Centre and Eli Lilly and Company, USA.

\section{References}

1. Groop LC (1992) Sulfonylureas in NIDDM. Diabetes Care 15:737-754

2. Matthews DR, Cull CA, Stratton IM, Holman RR, Turner RC, UK Prospective Diabetes Study (UKPDS) Group (1998) UKPDS 26: sulphonylurea failure in non-insulin-dependent diabetic patients over six years. Diabet Med 15:297-303

3. Turner RC (1998) The U.K. prospective diabetes study: a review. Diabetes Care 21(Suppl 3):C35-C38

4. UK Prospective Diabetes Study Group (1995) UK prospective diabetes study 16. Overview of 6 years' therapy of type II diabetes: a progressive disease. Diabetes 44:1249-1258

5. Turner RC, Cull CA, Frighi V, Holman RR, the UK Prospective Diabetes Study (UKPDS) Group (1999) Glycemic control with diet, sulfonylurea, metformin, or insulin in patients with type 2 diabetes mellitus: progressive requirement for multiple therapies (UKPDS 49). JAMA 281:2005-2012

6. Aronoff S, Rosenblatt S, Braithwaite S, Egan JW, Mathisen AL, Schneider RL, the Pioglitazone 001 Study Group (2000) Pioglitazone hydrochloride monotherapy improves glycemic control in the treatment of patients with type 2 diabetes: a 6-month randomized placebo-controlled dose-response study. Diabetes Care 23:1605-1611

7. Herz M, Johns D, Reviriego J et al and the GLAB Study Group (2003) A randomized, double-blind, placebo-controlled, clinical trial of the effects of pioglitazone on glycemic control and dyslipidemia in oral antihyperglycemic medication-naïve patients with type 2 diabetes mellitus. Clin Ther 25:1074-1095

8. Pavo I, Jermendy G, Varkonyi TT et al (2003) Effect of pioglitazone compared with metformin on glycemic control and indicators of insulin sensitivity in recently diagnosed patients with type 2 diabetes. J Clin Endocrinol Metab $88: 1637-1645$

9. Rosenblatt S, Miskin B, Glazer NB, Prince MJ, Robertson KE, Pioglitazone 026 Study Group (2001) The impact of pioglitazone on glycemic control and atherogenic dyslipidemia in patients with type 2 diabetes mellitus. Coron Artery Dis 12:413-423

10. Charbonnel BH, Matthews DR, Schernthaner G, Hanefeld M, Brunetti P on behalf of the QUARTET Study Group (2005) A long-term comparison of pioglitazone and gliclazide in patients with type 2 diabetes mellitus: a randomized, double-blind, parallel-group comparison trial. Diabet Med 22:399-405

11. Schernthaner G, Matthews DR, Charbonnel B, Hanefeld M, Brunetti P, the Quartet Study Group (2004) Efficacy and safety of pioglitazone versus metformin in patients with type 2 diabetes mellitus: a double-blind, randomized trial. J Clin Endocrinol Metab 89:6068-6076

12. Kipnes MS, Krosnick A, Rendell MS, Egan JW, Mathisen AL, Schneider RL (2001) Pioglitazone hydrochloride in combination with sulfonylurea therapy improves glycemic control in patients with type 2 diabetes mellitus: a randomized, placebocontrolled study. Am J Med 111:10-17

13. Einhorn D, Rendell M, Rosenzweig J, Egan JW, Mathisen AL, Schneider RL, the Pioglitazone 027 Study Group (2000) Pioglitazone hydrochloride in combination with metformin in the treatment of type 2 diabetes mellitus: a randomized, placebocontrolled study. Clin Ther 22:1395-1409 
14. Derosa G, Cicero AFG, Gaddi A et al (2004) Metabolic effects of pioglitazone and rosiglitazone in patients with diabetes and metabolic syndrome treated with glimepiride: a twelve-month, multicenter, double-blind, randomized, controlled, parallelgroup trial. Clin Ther 26:744-754

15. Rendell MS, Glazer NB, Ye Z (2003) Combination therapy with pioglitazone plus metformin or sulfonylurea in patients with Type 2 diabetes: influence of prior antidiabetic drug regimen. J Diabetes Complications 17:211-217

16. Hanefeld M, Brunetti P, Schernthaner GH, Matthews DR, Charbonnel $\mathrm{BH}$, on behalf of the QUARTET Study Group (2004) One-year glycemic control with a sulfonylurea plus pioglitazone versus a sulfonylurea plus metformin in patients with Type 2 diabetes. Diabetes Care 27:141-147

17. Matthews DR, Charbonnel BH, Hanefeld M, Brunetti P, Schernthaner G (2005) Long-term therapy with addition of pioglitazone to metformin compared with the addition of gliclazide to metformin in patients with type 2 diabetes: a randomized, comparative study. Diabetes/Metab Res Rev 21: 167-174

18. Dobiášová M, Frohlich J (2001) The plasma parameter log (TG/HDL-C) as an atherogenic index: correlation with lipoprotein particle size and esterification rate in apoB-lipoproteindepleted plasma $\left(\mathrm{FER}_{\mathrm{HDL}}\right)$. Clin Biochem 34:583-588

19. Johnson M, Krosnick A, Carson P, McDade AM, Laraway K (1998) A retrospective chart review of uncontrolled use of metformin as an add-on therapy in type 2 diabetes. Clin Ther 20 : 691-698

20. DeFronzo RA, Goodman AM, Multicenter Metformin Study Group (1995) Efficacy of metformin in patients with noninsulin-dependent diabetes mellitus. N Engl J Med 333:541549

21. Charpentier G, Fleury F, Kabir M, Vaur L, Halimi S (2001) Improved glycaemic control by addition of glimepiride to metformin monotherapy in type 2 diabetic patients. Diabet Med 18:828-834

22. Ohkubo Y, Kishikawa H, Araki E et al (1995) Intensive insulin therapy prevents the progression of diabetic microvascular complications in Japanese patients with non-insulin-dependent diabetes mellitus: a randomized prospective 6-year study. Diabetes Res Clin Pract 28:103-117

23. Stratton IM, Adler AI, Neil AW et al, on behalf of the UK Prospective Diabetes Study Group (2000) Association of glycaemia with macrovascular and microvascular complications of type 2 diabetes (UKPDS 35): prospective observational study. BMJ 321:405-412

24. Dunn CJ, Peters DH (1995) Metformin: a review of its pharmacological properties and therapeutic use in non-insulin-dependent diabetes mellitus. Drugs 49:721-749
25. UK Prospective Diabetes Study Group (1998) UKPDS 28: a randomized trial of efficacy of early addition of metformin in sulfonylurea-treated type 2 diabetes. Diabetes Care 21:87-92

26. Taskinsen M-R (2003) LDL-cholesterol, HDL-cholesterol or triglycerides-which is the culprit? Diabetes Res Clin Pract 61: S19-S26

27. Fontbonne A, Eschwège E, Cambien F et al (1989) Hypertriglyceridaemia as a risk factor of coronary heart disease mortality in subjects with impaired glucose tolerance or diabetes: results from the 11-year follow-up of the Paris Prospective Study. Diabetologia 32:300-304

28. West KM, Ahuja MMS, Bennett PH et al (1983) The role of circulating glucose and triglyceride concentrations and their interactions with other "risk factors" as determinants of arterial disease in nine diabetic population samples from the WHO multinational study. Diabetes Care 6:361-369

29. Wilson PWF, Kannel WB, Anderson KM (1985) Lipids, glucose intolerance and vascular disease: the Framingham Study. Monogr Atheroscler 13:1-11

30. Lehto S, Rönnemaa T, Haffner SM, Pyörälä K, Kallio V, Laakso M (1997) Dyslipidemia and hyperglycemia predict coronary heart disease events in middle-aged patients with NIDDM. Diabetes 46:1354-1359

31. Austin MA, Breslow JL, Hennekens CH, Buring JE, Willett WC, Krauss RM (1988) Low-density lipoprotein subclass patterns and risk of myocardial infarction. JAMA 260:1917-1921

32. Campos H, Genest JJ Jr, Blijlevens E et al (1992) Low density lipoprotein particle size and coronary artery disease. Arterioscler Thromb 12:187-195

33. Coresh J, Kwiterovich PO Jr, Smith HH, Bachorik PS (1993) Association of plasma triglyceride concentration and LDL particle diameter, density, and chemical composition with premature coronary artery disease in men and women. J Lipid Res 34:1687-1697

34. Gardner CD, Fortmann SP, Krauss RM (1996) Association of small low-density lipoprotein particles with the incidence of coronary artery disease in men and women. JAMA 276:875881

35. Feingold KR, Grunfeld C, Pang M, Doerrler W, Krauss RM (1992) LDL subclass phenotypes and triglyceride metabolism in non-insulin-dependent diabetes. Arterioscler Thromb 12 : 1496-1502

36. Charbonnel B, Dormandy J, Erdmann E, Massi-Benedetti M, Skene A (2004) The Prospective Pioglitazone Clinical Trial in Macrovascular Events (PROactive): can pioglitazone reduce cardiovascular events in diabetes? Study design and baseline characteristics of 5,238 patients. Diabetes Care 27:1647-1653 